\title{
Process-controllable modulation of plasmon-induced transparency in terahertz metamaterials
}

\author{
Hao Sun (孙 豪) $)^{1, \dagger}$ ，Jie Yang (杨 杰 $)^{2, \dagger}$, Hengzhu Liu (刘衡竹) $)^{1}$, Dan Wu (吴 丹 $)^{3}$, and Xin Zheng (郑 金金) \\ ${ }^{1}$ College of Computer, National University of Defense Technology, Changsha 410073, China \\ ${ }^{2}$ National Innovation Institute of Defense Technology, Beijing 100010, China \\ ${ }^{3}$ Graduate School, National University of Defense Technology, Changsha 410073, China
}

*Corresponding author: zhengxin@nudt.edu.cn

Received July 8, 2020 | Accepted September 4, 2020 | Posted Online November 26, 2020

\begin{abstract}
Recently reported plasmon-induced transparency (PIT) in metamaterials endows the optical structures in classical systems with quantum optical effects. In particular, the nonreconfigurable nature in metamaterials makes multifunctional applications of PIT effects in terahertz communications and optical networks remain a great challenge. Here, we present an ultrafast process-selectable modulation of the PIT effect. By incorporating silicon islands into diatomic metamaterials, the PIT effect is modulated reversely, depending on the vertical and horizontal configurations, with giant modulation depths as high as $129 \%$ and $109 \%$. Accompanied by the enormous switching of the transparent window, remarkable slow light effect occurs.
\end{abstract}

Keywords: terahertz metamaterials; ultrafast photoswitching; plasmon-induced transparency; all-optical modulation. DOI: 10.3788/COL202119.013602

\section{Introduction}

In an atomic system, quantum coherence has resulted in interesting and abundant outcomes, including trapping of atoms, Bose-Einstein condensates, and laser cooling. As an intriguing instance of quantum coherence, electromagnetically induced transparency (EIT) is a phenomenon where a non-transparent medium is changed into a transparent one with a narrow window, leading to the dynamical storage of light in solid-state systems ${ }^{[1]}$ and no inversion of the laser ${ }^{[2]}$. In a three-level atomic system, two excitation ways toward high energy level destructively interfere with each other, which leads to a cancellation of absorptions ${ }^{[3,4]}$. Owing to the high quality factor and strong dispersion, the EIT phenomenon has demonstrated excellent potentials for intriguing photonic applications, such as nonlinear and slow light devices. In classical systems, it has been theoretically proved that the interference between coherent optical waves can induce an EIT-like phenomenon ${ }^{[5]}$, which can be exploited to block and capture light ${ }^{[6]}$. On account of the negligible propagation loss, mimicking the EIT phenomenon is promising and attractive in metamaterials. Notably, metamaterials are novel artificial metal structures that are composed of periodically arranged basic unit cells, which exhibit response to the electric and magnetic components of the electromagnetic field ${ }^{[7]}$. By manually designing the geometric parameters, it is feasible to tailor the propagation characteristics with a large degree of flexibility and intrigue numerous interesting properties, including enhanced non-linearity and tremendous group refractive index ${ }^{[8,9]}$, whose schemes have been applied on optical buffers $^{[10]}$, slow light devices ${ }^{[11,12]}$, and biochemical sensors ${ }^{[13]}$. Particularly in the terahertz (THz) field, the metamaterials serve as distinguished candidates for sensing ${ }^{[14]}$, next-generation communication $^{[15]}$, and imaging applications ${ }^{[16]}$.

Several essential units are commonly utilized in constructing $\mathrm{THz}$ metamaterials, such as cut-wire resonator ${ }^{[17]}$, closed-ring resonator $(\mathrm{CRR})^{[18]}$, and split-ring resonator (SRR) ${ }^{[19]}$, allowing for prosperous applications relative to the plasmonic hybridization ${ }^{[20]}$. In practical experiments, EIT-like phenomenon in metamaterials is induced and observed by exciting a polaritonic resonance formed by bright and dark eigenmodes $^{[21]}$. Precisely, the dark mode often originates from the broken symmetry of system, such as SRRs. In general, the bright eigenmode needs an absorption of the electric field with a broad width, whereas the dark eigenmode presents a narrow line width $^{[22]}$. Similar to the EIT phenomenon, the interference between bright and dark eigenmodes can be regarded as a plasmonic analogue of the EIT phenomenon, which is plasmoninduced transparency (PIT) in classical systems ${ }^{[23]}$. In order to enhance the active control of the PIT phenomenon by external stimuli, the metamaterials are often hybridized with a variety of materials, including superconductors ${ }^{[24]}$, semiconductors ${ }^{[25]}$, and graphene ${ }^{[26-29]}$, using their natural 
response to electrical ${ }^{[30,31]}$, optical ${ }^{[32]}$, mechanical ${ }^{[33]}$, and thermal stimuli ${ }^{[34]}$. Among these conductors, semiconductors can provide carrier injection to shunt the capacity in metamaterials, controlled by external stimuli. One salient and significant modulating approach is the all-optical modulation of the PIT effect in a picosecond time scale, which is highly desired to novel ultrafast devices ${ }^{[35-40]}$. To date, all-optical modulation of metamaterials and semiconductors has been established as a hot topic worthy of numerous scholar attentions ${ }^{[41,42]}$. However, most of the reported works focus on a single PIT modulation, in which only one function can be acquired at a time.

In this work, we present a detailed investigation on the simultaneous forward and reverse modulation of the PIT phenomenon along two polarization directions, respectively, in a delicately designed metamaterial device. In this device, SRRs and split cut-wire resonators (SCWRs) are fabricated on a silicon-on-sapphire (SOS) wafer. Accounting for the different metal structures along two planar perpendicular directions, the PIT phenomena are simultaneously modulated and demodulated in two directions via external optical stimuli. Importantly, one can actively manipulate and select the modulating process by rotating the metadevice. The optical-pump THz-probe (OPTP) measurements reveal that the modulation of PIT phenomena is accompanied by the sharp transparent peaks with modulation depths of $109 \%$ and $129 \%$ and remarkable slow light effects. Alternatively, the response time is measured to be less than 2 ps, owing to the ultrafast response of $\mathrm{Si}$.

\section{Results and Discussion}

The schematic of the metamaterial device for OPTP is depicted in Fig. 1(a). THz beams travel through the area on the surface of metamaterials, where the laser beam illuminates on Si to excite the free carriers. Specifically, the THz beams are linearly polarized along two planar perpendicular directions, denoted as $x$

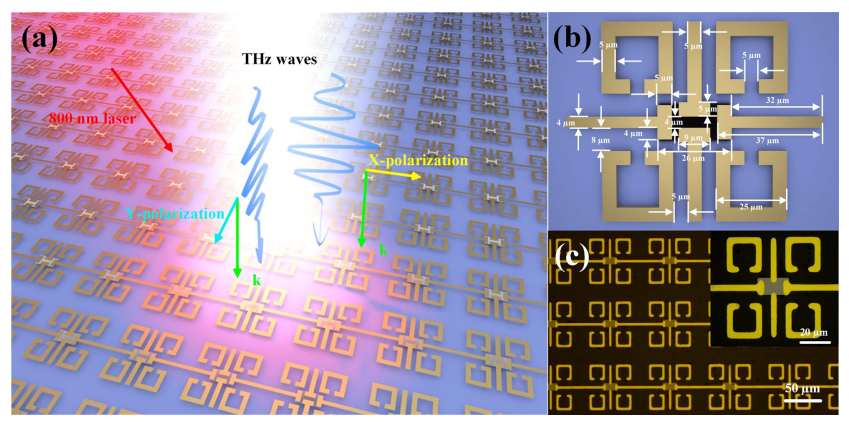

Fig. 1. (a) Diagrammatic illustration of the polarization-dependent PIT modulation in the metadevice for OPTP spectroscopy. (b) Schematic of the unit cell of the metadevice. The sizes have been marked in the figure. The black area is the bared Si island, whose sizes are $26 \mu \mathrm{m}$ in the horizontal direction and $12 \mu \mathrm{m}$ in the vertical direction, respectively. (c) Optical microscopic image of the periodically arranged unit cells. Inset is an amplified photo of a single unit cell. The periods are $90 \mu \mathrm{m}$ and $95 \mu \mathrm{m}$ in directions of $x$ polarization and $y$ polarization, respectively. polarization and $y$ polarization, respectively. The configuration of the unit cell is displayed in Fig. 1(b). It is seen from this figure that the golden structure is deposited onto the surface of an SOS wafer, while the Si layer is sculptured into two parts, which includes the shape of islands and the metal structures, in order to electrically connect the SCWRs of metamaterials. In addition, the fabricated metadevice is in great agreement with the designed layout, which is indicated by the optical microscopic image in Fig. 1(c).

To begin with, the polarization dependence of $\mathrm{THz}$ waves transmission rates in the metaphotonic device is investigated, with the results being presented in Fig. 2. In case of THz $x$ polarization, an intrinsic PIT phenomenon is observed in the frequency regime from $0.4 \mathrm{THz}$ to $1.2 \mathrm{THz}$ without an outer laser pump, represented by the black curve in Fig. 2(a). Moreover, a sharp transparent window appeared to be $86.3 \%$ at $0.81 \mathrm{THz}$, between a pair of dips at $0.70 \mathrm{THz}$ and $1.03 \mathrm{THz}$, with a quality factor of 4.0. When under an exterior laser pump excitation with its power changing from $0 \mathrm{~mW} / \mathrm{cm}^{2}$ to $2000 \mathrm{~mW} / \mathrm{cm}^{2}$, the PIT phenomenon in this metaphotonic device gradually disappears. On the other hand, for $\mathrm{THz} y$ polarization, a small transparent dip is found at $0.91 \mathrm{THz}$ without an external laser pump, associated with a high transmission rate, which is shown as the black curve in Fig. 2(b). Pumped by laser beams, the metaphotonic device realized a PIT effect. One significant finding is that when the laser pump ascends from $0 \mathrm{~mW} / \mathrm{cm}^{2}$ to $2000 \mathrm{~mW} / \mathrm{cm}^{2}$, the quality factor gradually increases to 4.7. Alternatively, the sharpest transparent window appears in between two other dips. The numerically calculated transmission rates are shown in Figs. 2(c) and 2(d). In order to mimic the variational conductivity of the Si island pumped by
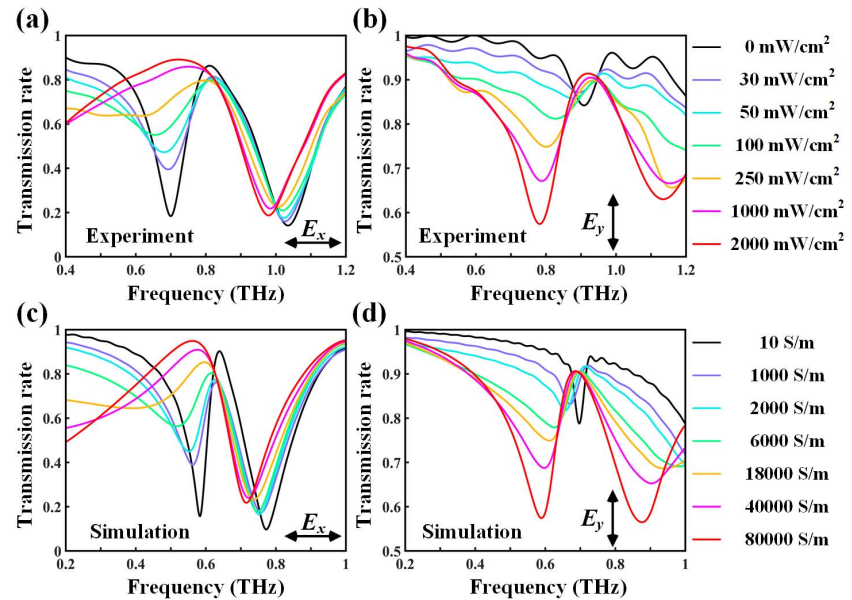

Fig. 2. Polarization-dependent modulation of PIT effects in two perpendicular $\mathrm{THz}$ polarization directions, with a time delay of $40 \mathrm{ps}$. Experimentally measured transmission rates of THz waves for (a) $x$ polarization and (b) y polarization of metaphotonic devices, pumped by a series of pumping powers (shown as labels). The numerically simulated transmission rates of $\mathrm{THz}$ waves for (c) x polarization and (b) y polarization of metaphotonic devices, with conductivity of Si island varying corresponding to the experimental measurement (shown as labels). 
laser beams, the conductivity of $\mathrm{Si}$ in calculating was manually set from $10 \mathrm{~S} / \mathrm{m}$ to $80,000 \mathrm{~S} / \mathrm{m}$. Considering the intrinsic conductivity of $\mathrm{Si}$, the minimum of its conductivity was set as $10 \mathrm{~S} / \mathrm{m}$ rather than $0 \mathrm{~S} / \mathrm{m}$. Importantly, the shapes of calculated transmission rates are consistent with the measured data. However, the red-shift behaviors are discovered from the calculated transmission rates when compared with the experimentally measured spectra. One reasonable explanation is that the fabrication is deviated from the designed layout. This error causes the electromagnetic resonances appearing at blue-shifted frequencies. With the conductivity set manually, the trend of calculated transmission rates matches well with that of experimental measurement. The difference between transmission rates of the Fano peak and the left Fano dip is defined as the maximum of transmission difference $\left(D_{M}\right)$ when the PIT effect appears. In the case that the PIT effect vanishes, the difference of transmission rates is defined as the minimum of transmission difference $\left(D_{m}\right)$. In this context, the modulation depth is defined as $\left(D_{M}-D_{m}\right) / D_{M}$. According to the experimental measurements, the modulation depths are extracted to be $109 \%$ for $\mathrm{THz} x$ polarization and $129 \%$ for $\mathrm{THz} y$ polarization.

Additional insights are furnished by the exploration of the slow light property in metaphotonic devices. Here, both the numerical simulations and experimental measurements are performed to acquire the $\mathrm{THz}$ transient group delay, whose results are demonstrated in Fig. 3. In cases of THz $x$ polarization, a pair of dips appear at $0.70 \mathrm{THz}$ and $1.04 \mathrm{THz}$ when under the pump fluence of $0 \mathrm{~mW} / \mathrm{cm}^{2}$, shown as the black curve in Fig. 3(a), whose group delays are $-7.9 \mathrm{ps}$ and $-5.1 \mathrm{ps}$, respectively. In term of the $y$ polarization [see Fig. 3(b)], the PIT phenomenon occurs when the metadevice is pumped at $2000 \mathrm{~mW} / \mathrm{cm}^{2}$, where a pair of dips associated with delay values of $-1.22 \mathrm{ps}$ and -0.49 ps appear at $0.78 \mathrm{THz}$ and $1.13 \mathrm{THz}$, respectively. Additionally, only one dip of -0.43 ps is observed at $0.91 \mathrm{THz}$
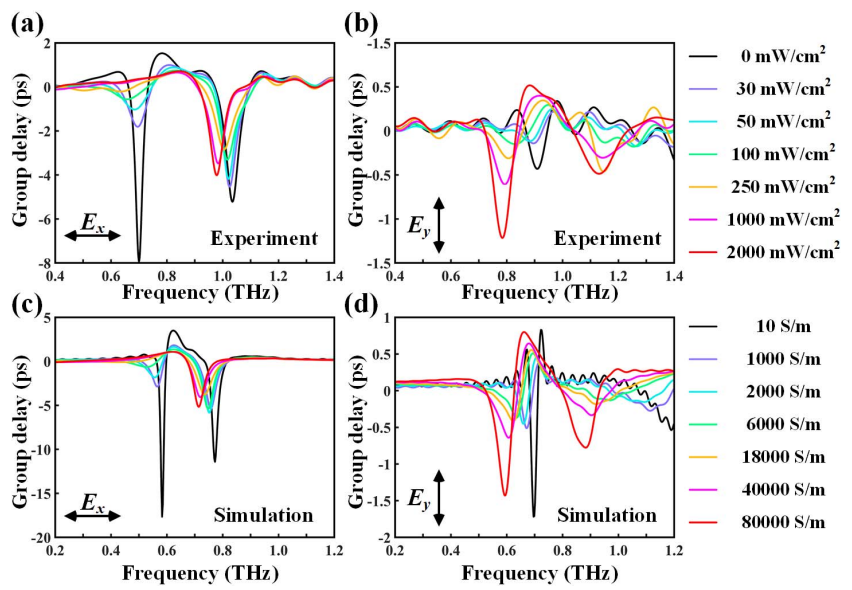

Fig. 3. Experimentally measured THz transient group delay for (a) x polarization and (b) $y$ polarization, with the metaphotonic device pumped by laser beams for a series of fluence (as labels). Numerically calculated group delay of THz waves for (c) xpolarization and (d) y polarization with conductivity of $\mathrm{Si}$ island changing as labels. The time delay was 40 ps. when the laser pump is turned off. Moreover, the numerical calculations are demonstrated in Figs. 3(c) and 3(d), which are in good agreement with the experimental measurements, except for the two following aspects. Firstly, the dips for $\mathrm{THz}$ $x$ polarization reach as high as $-17.66 \mathrm{ps}$ and $-11.41 \mathrm{ps}$, and their shapes are sharper than the experimentally measured ones. In addition, for $y$ polarization, when conductivity is $10 \mathrm{~S} / \mathrm{m}$, the dip of the numerical calculation is also sharper than experimental measurements. However, other dips are very consistent with experiments, without the presence of the above issues. It is referred that the precision of the experimental measurement depends on the minimum movement of the translational stage. As a result, the fixed space interval of the translational stage causes the sharp dips that cannot be detected. Secondly, the frequency of calculated results shows red-shifting features when compared with the measured ones, which may be caused by the mismachining tolerance.

In addition to capturing the influence of diverse pump fluence on modulation, the transient process of modulation in the time domain was also studied, as presented in Fig. 4. When under a pump with fluence of $2000 \mathrm{~mW} / \mathrm{cm}^{2}$, the PIT phenomenon occurs, accompanied by a transparent window. This PIT phenomenon vanishes with an increasing time delay, as illustrated in Fig. 4(a). In the meantime, the transparent peak and the right transmission dip both show red shifts. The time delay is defined as $0 \mathrm{ps}$ for the translational stage at the original state. At this time, the $\mathrm{THz}$ waves arrive earlier than laser pump. With the increment of time delay, the intrinsic transparent dip reappears, and other transparent peaks and dips exhibit blue shifts towards their original position. In particular, when excited by a pump with fluence of $2000 \mathrm{~mW} / \mathrm{cm}^{2}$, the PIT effect suddenly occurs [see Fig. 4(b)]. A pair of transparent dips appear, accompanied by a transparent peak. As time delay further increases, the PIT effect gradually vanishes, and the transmission rates recover towards the original spectrum.

To investigate the optical characteristic of the SOS wafer, OPTP experiments are carried out using the $\mathrm{THz}$ timedomain system (TDS). Here, the laser pumps with fluences of $500 \mathrm{~mW} / \mathrm{cm}^{2}, 1000 \mathrm{~mW} / \mathrm{cm}^{2}$, and $2000 \mathrm{~mW} / \mathrm{cm}^{2}$ were applied to spots on the SOS wafer. The dynamics of the SOS wafer is described by the transient negative differential transmission $(-\Delta T / T)$. The transient negative differential transmission indicates the real-time dynamics of photo-generated carriers
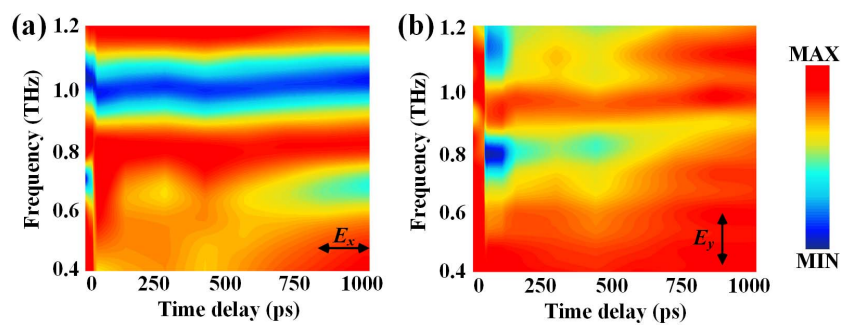

Fig. 4. Experimentally measured $\mathrm{THz}$ transient color map of transmission rates for (a) $x$ polarization and (b) y polarization, under pump fluence of $2000 \mathrm{~mW} / \mathrm{cm}^{2}$. 


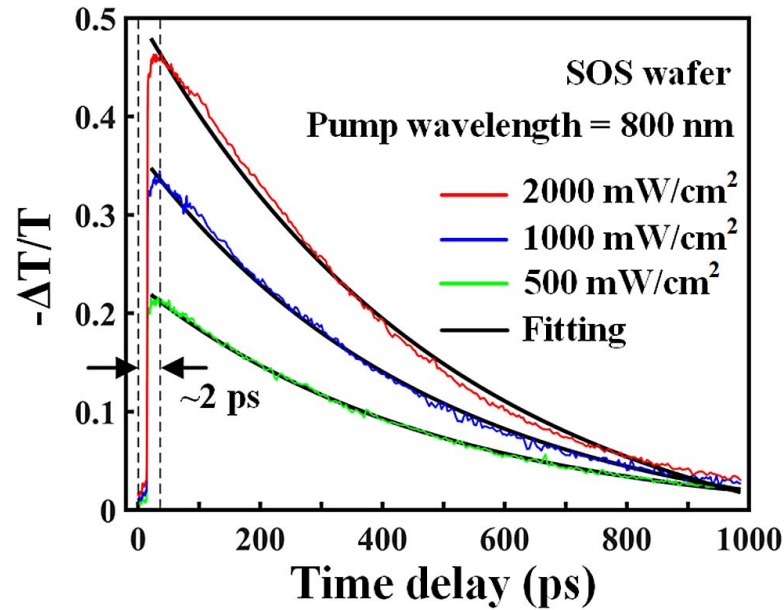

Fig. 5. Negative differential transmission of SOS wafer pumped by laser beams at a series of powers (as labels). The measured data was fitted using a single exponential function.

of the SOS wafer, whose peak is proportional to the photo-conductivity, assuming that the carrier mobilities are unchanged ${ }^{[43]}$. As shown in Fig. 5, the photo-generated carriers reach the maximal value within $\sim 2$ ps. Limited by the total length of translational stages, the whole period of photo-generated carriers containing the exciting and decaying processes could not be completely recorded. To analyze the temporal evolution, a single exponential function $\left[-\Delta T(t) / T_{0}=A e^{-t / \tau}+B\right]$ is exploited to fit the experimentally measured results, under an assumption that the slow decay is attributed to trap-assisted recombination. Thus, the fitting curves are shown in Fig. 5, with the decay constants retrieved from the fitting curves being 473 ps at $1000 \mathrm{~mW} / \mathrm{cm}^{2}$.

Aiming at demonstrating the underlying mechanism of formation and vanishing, we performed numerical calculations on transmission rates and electromagnetic field distributions of the units in metaphotonic devices. The calculated results are shown in Fig. 6. The orthogonal SCWRs are designed for the modulation in vertical directions. For $\mathrm{THz} x$ polarization, with conductivity of $\mathrm{Si}$ set as $10 \mathrm{~S} / \mathrm{m}$, two transparent dips appear when there are only SRR pairs and CW, respectively. The localized surface plasmon (LSP) resonance in SCWRs and the inductive-capacitive (LC) resonance in SRR pairs couple with each other, leading to a typical PIT phenomenon with a transparent window between a pair of transparent dips. The electromagnetic field of these three units is shown in Fig. 6(e). With conductivity of $\mathrm{Si}$ set as $80,000 \mathrm{~S} / \mathrm{m}$, the LSP resonance in the SCWR disappears, since the effective electrical length of the SCWR is changed by the Si island. The LC resonance alone is not able to excite the PIT phenomenon. Consequently, the PIT phenomenon vanishes in the meantime, as shown in Fig. 6(f). For the $\mathrm{THz} y$ polarization, when the conductivity is set as $10 \mathrm{~S} / \mathrm{m}$, the LSP resonance is vibrated in two parts of SCWRs, as shown in Fig. 6(g), corresponding to a transparent dip at around $1.27 \mathrm{THz}$ illustrated as the inset in Fig. 6(c). In other aspects, the transmission rate of SRR is at a high level, resulting
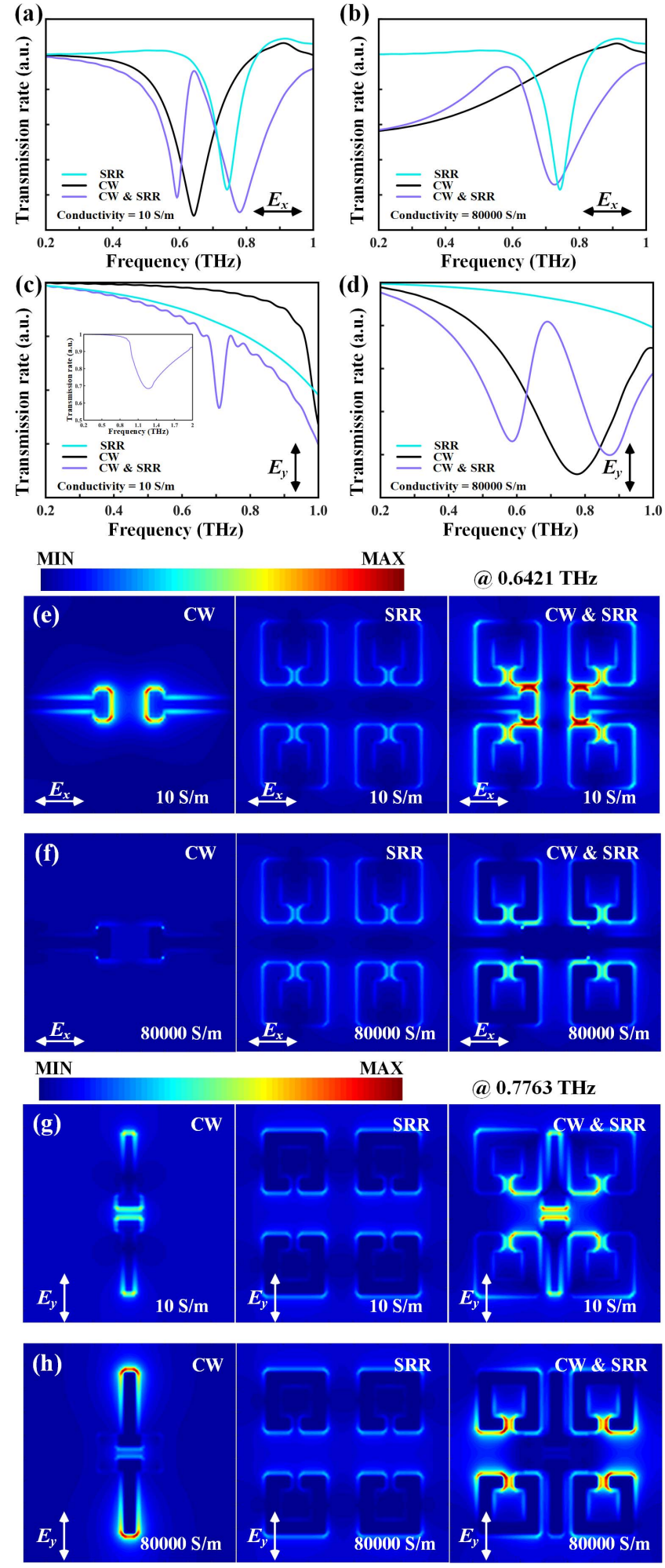

Fig. 6. Intrinsic coupling of the PIT effect for appearing and vanishing. The relative transmission rate of SRR, CW, and their combination, respectively, with conductivity of Si set as (a) $10 \mathrm{~S} / \mathrm{m}$ and (b) 80,000 S/m for THz $x$ polarization, and (c) $10 \mathrm{~S} / \mathrm{m}$ and (d) $80,000 \mathrm{~S} / \mathrm{m}$ for THz y polarization. Numerically calculated electromagnetic field distributions of these units with conductivity of (e) $10 \mathrm{~S} / \mathrm{m}$, (f) $80,000 \mathrm{~S} / \mathrm{m}$ for THz x polarization (at $0.6421 \mathrm{THz}$ ), and (g) $10 \mathrm{~S} / \mathrm{m}$, (h) 80,000 S/m for $\mathrm{THz} y$ polarization (at $0.7763 \mathrm{THz}$ ). 
in the occurrence of a transparent dip at $0.71 \mathrm{THz}$ in units of SCWR and SRR. As conductivity of $\mathrm{Si}$ increases to $80,000 \mathrm{~S} / \mathrm{m}$, the LSP resonance is in the whole SCWR. The electromagnetic field of LSP resonance stimulates the LC resonance in SRR. Then, these two resonances couple with each other, resulting in the PIT phenomenon. The formation process is shown in Figs. 6(d) and 6(h).

\section{Conclusion}

In summary, we have established a comprehensive study on the polarization-dependent modulation of the PIT phenomenon in the $\mathrm{THz}$ metaphotonic device. Specifically, the SCWRs and SRRs are fabricated on an SOS wafer of this device. The forward and reverse PIT modulating processes can be manipulated via the rotation of the metadevice. Moreover, remarkable modulation effects of transmission rate and group delay are observed, the modulation depths of which reach $129 \%$ and $109 \%$, respectively. Additionally, the resonant $\mathrm{THz}$ transmission is modulated on the picosecond time scale, owing to the ultrafast response of $\mathrm{Si}$. This work set out to gain a better understanding of actively controlling the modulating process of the PIT phenomenon, which also inspires more ingenious designs for novel photonics devices.

\section{Acknowledgement}

This work was supported by the National Natural Science Foundation of China (Nos. 11804387, 11802339, 11805276, 11902358, 61805282, and 61801498) and the Scientific Researches Foundation of National University of Defense Technology (Nos. ZK18-03-22, ZK18-01-03, and ZK18-03-36).

${ }^{\dagger}$ These authors contributed equally to this work.

\section{References}

1. J. J. Longdell, E. Fraval, M. J. Sellars, and N. B. Manson, "Stopped light with storage times greater than one second using EIT in a solid," Phys. Rev. Lett. 95, 063601 (2005).

2. L. V. Hau, S. E. Harris, Z. Dutton, and C. H. Behroozi, "Light speed reduction to 17 metres per second in an ultracold atomic gas," Nature 397, 594 (1999).

3. S. E. Harris, "Electromagnetically induced transparency," Phys. Today 50, 36 (1997).

4. A. Kasapi, M. Jain, G. Y. Yin, and S. E. Harris, "Electromagnetically induced transparency: propagation dynamics," Phys. Rev. Lett. 74, 2447 (1995).

5. Y. He, H. Zhou, Y. Jin, and S. He, "Plasmon induced transparency in a dielectric waveguide," Appl. Phys. Lett. 99, 043113 (2011).

6. C. L. G. Alzar, M. A. G. Martinez, and P. Nussenzveig, "Classical analog of electromagnetically induced transparency," Am. J. Phys. 70, 37 (2002).

7. X. Yang, M. Yu, D.-L. Kwong, and C. W. Wong, "All-optical analog to electromagnetically induced transparency in multiple coupled photonic crystal cavities," Phys. Rev. Lett. 102, 173902 (2009).

8. P. Tassin, L. Zhang, T. Koschny, E. N. Economou, and C. M. Soukoulis, "Low-loss metamaterials based on classical electromagnetically induced transparency," Phys. Rev. Lett. 102, 053901 (2009).
9. Q. Xu, S. Sandhu, M. L. Povinelli, J. Shakya, S. Fan, and M. Lipson, "Experimental realization of an on-chip all-optical analogue to electromagnetically induced transparency," Phys. Rev. Lett. 96, 123901 (2006).

10. N. Liu, L. Langguth, T. Weiss, J. Kästel, M. Fleischhauer, T. Pfau, and $\mathrm{H}$. Giessen, "Plasmonic analogue of electromagnetically induced transparency at the Drude damping limit," Nature Mater. 8, 758 (2009).

11. C. Liu, P. Liu, C. Yang, Y. Lin, and H. Liu, "Analogue of dual-controlled electromagnetically induced transparency based on a graphene metamaterial," Carbon 142, 354 (2019).

12. H. Jung, H. Jo, W. Lee, B. Kim, H. Choi, M. S. Kang, and H. Lee, "Electrical control of electromagnetically induced transparency by terahertz metamaterial funneling," Adv. Opt. Mater. 7, 1801205 (2019).

13. M. Liu, Z. Tian, X. Zhang, J. Gu, C. Ouyang, J. Han, and W. Zhang, "Tailoring the plasmon-induced transparency resonances in terahertz metamaterials," Opt. Express 25, 19844 (2017).

14. J. Gu, R. Singh, X. Liu, X. Zhang, Y. Ma, S. Zhang, S. A. Maier, Z. Tian, A. K. Azad, H.-T. Chen, A. J. Taylor, J. Han, and W. Zhang, "Active control of electromagnetically induced transparency analogue in terahertz metamaterials," Nat. Commun. 3, 1151 (2012).

15. R. Singh, I. A. I. Al-Naib, Y. Yang, D. R. Chowdhury, W. Cao, C. Rockstuhl, T. Ozaki, R. Morandotti, and W. Zhang, "Observing metamaterial induced transparency in individual Fano resonators with broken symmetry," Appl. Phys. Lett. 99, 201107 (2011).

16. X. Yin, T. Feng, S. Yip, Z. Liang, A. Hui, J. C. Ho, and J. Li, "Tailoring electromagnetically induced transparency for terahertz metamaterials: from diatomic to triatomic structural molecules," Appl. Phys. Lett. 103, 021115 (2013).

17. A. A. Yanik, A. E. Cetin, M. Huang, A. Artar, S. H. Mousavi, A. Khanikaev, J. H. Connor, G. Shvets, and H. Altug, "Seeing protein monolayers with naked eye through plasmonic Fano resonances," Proc. Natl. Acad. Sci. 108, 11784 (2011).

18. N. Liu, M. Hentschel, T. Weiss, A. P. Alivisatos, and H. Giessen, "Threedimensional plasmon rulers," Science 332, 1407 (2011).

19. Z.-G. Dong, H. Liu, J.-X. Cao, T. Li, S.-M. Wang, and X. Zhang, "Enhanced sensing performance by the plasmonic analogue of electromagnetically induced transparency in active metamaterials," Appl. Phys. Lett. 97, 114101 (2010).

20. R. Taubert, M. Hentschel, J. Kästel, and H. Giessen, "Classical analog of electromagnetically induced absorption in plasmonics," Nano Lett. 12, 1367 (2012).

21. R. Schittny, M. Kadic, T. Buckmann, and M. Wegener, "Invisibility cloaking in a diffusive light scattering medium," Science 345, 427 (2014).

22. J. B. Pendry, "Negative refraction makes a perfect lens," Phys. Rev. Lett. 85, 3966 (2000).

23. D. Lu and Z. Liu, "Hyperlenses and metalenses for far-field super-resolution imaging," Nat. Commun. 3, 1205 (2012).

24. X. Tian and Z.-Y. Li, "Visible-near infrared ultra-broadband polarization-independent metamaterial perfect absorber involving phase-change materials," Photon. Res. 4, 146 (2016).

25. H. Jung, J. Koo, E. Heo, B. Cho, C. In, W. Lee, H. Jo, J. H. Cho, H. Choi, M. S. Kang, and H. Lee, "Electrically controllable molecularization of terahertz meta-atoms," Adv. Mater. 30, 1802760 (2018).

26. M. M. Jadidi, A. B. Sushkov, R. L. Myers-Ward, A. K. Boyd, K. M. Daniels, D. K. Gaskill, M. S. Fuhrer, H. D. Drew, and T. E. Murphy, "Tunable terahertz hybrid metal-graphene plasmons," Nano Lett. 15, 7099 (2015).

27. Z. Chen, X. Chen, L. Tao, K. Chen, M. Long, X. Liu, K. Yan, R. I. Stantchev, E. Pickwell-MacPherson, and J.-B. Xu, "Graphene controlled Brewster angle device for ultra broadband terahertz modulation," Nat. Commun. 9, 4909 (2018).

28. H.-T. Chen, J. F. O'Hara, A. K. Azad, A. J. Taylor, R. D. Averitt, D. B. Shrekenhamer, and W. J. Padilla, "Experimental demonstration of frequency-agile terahertz metamaterials," Nat. Photon. 2, 295 (2008).

29. Y. Fan, N.-H. Shen, F. Zhang, Q. Zhao, Z. Wei, P. Zhang, J. Dong, Q. Fu, $\mathrm{H}$. Li, and C. M. Soukoulis, "Photoexcited graphene metasurfaces: significantly enhanced and tunable magnetic resonances," ACS Photon. 5, 1612 (2018).

30. D. Shrekenhamer, J. Montoya, S. Krishna, and W. J. Padilla, "Four-color Metamaterial absorber THz spatial light modulator," Adv. Opt. Mater. 1, 905 (2013). 
31. H. Jung, J. Koo, E. Heo, B. Cho, C. In, W. Lee, H. Jo, J. H. Cho, H. Choi, M. S. Kang, and H. Lee, "Electrically controllable molecularization of terahertz meta-atoms," Adv. Mater. 30, 1802760 (2018).

32. S. Sim, H. Jang, N. Koirala, M. Brahlek, J. Moon, J. H. Sung, J. Park, S. Cha, S. Oh, M.-H. Jo, J.-H. Ahn, and H. Choi, "Ultra-high modulation depth exceeding $2400 \%$ in optically controlled topological surface plasmons," Nat. Commun. 6, 8814 (2015).

33. W. M. Zhu, A. Q. Liu, T. Bourouina, D. P. Tsai, J. H. Teng, X. H. Zhang, G. Q. Lo, D. L. Kwong, and N. I. Zheludev, "Microelectromechanical Maltese-cross metamaterial with tunable terahertz anisotropy," Nat. Commun. 3, 1274 (2012).

34. M. Liu, H. Y. Hwang, H. Tao, A. C. Strikwerda, K. Fan, G. R. Keiser, A. J. Sternbach, K. G. West, S. Kittiwatanakul, J. Lu, S. A. Wolf, F. G. Omenetto, X. Zhang, K. A. Nelson, and R. D. Averitt, "Terahertzfield-induced insulator-to-metal transition in vanadium dioxide metamaterial," Nature 487, 345 (2012).

35. Y. Hu, T. Jiang, J. Zhou, H. Hao, H. Sun, H. Ouyang, M. Tong, Y. Tang, H. Li, J. You, X. Zheng, Z. Xu, and X. Cheng, "Ultrafast terahertz frequency and phase tuning by all-optical molecularization of metasurfaces," Adv. Opt. Mater. 7, 1901050 (2019).

36. Y. Hu, T. Jiang, J. Zhou, H. Hao, H. Sun, H. Ouyang, M. Tong, Y. Tang, H. Li, J. You, X. Zheng, Z. Xu, and X. Cheng, "Ultrafast terahertZ transmission/ group delay switching in photoactive $\mathrm{WSe}_{2}$-functionalized metaphotonic devices," Nano Energy 7, 994 (2019).
37. H. Sun, Y. Hu, Y. Tang, J. You, J. Zhou, H. Liu, and X. Zheng, "Ultrafast polarization-dependent all-optical switching of germanium-based metaphotonic devices," Photon. Res. 8, 263 (2020).

38. H. Sun, Y. Tang, Y. Hu, J. You, H. Liu, and X. Zheng, "Ultrafast polarizationdependent all-optical switching of germanium-based metaphotonic devices," Chin. Opt. Lett. 18, 092402 (2020).

39. J. Zhou, Y. Hu, T. Jiang, H. Ouyang, H. Li, Y. Sui, H. Hao, J. You, X. Zheng, $\mathrm{Z}$. $\mathrm{Xu}$, and $\mathrm{X}$. Cheng, "Ultrasensitive polarization-dependent terahertz modulation in hybrid perovskites plasmon-induced transparency devices," Photon. Res. 7, 994 (2019).

40. J. Zhou, C. Zhang, Q. Liu, J. You, X. Zheng, X. Cheng, and T. Jiang, "Controllable all-optical modulation speed in hybrid silicon-germanium devices utilizing the electromagnetically induced transparency effect," Nanophotonics 9, 2797 (2020).

41. Y. Hu, T. Jiang, H. Sun, M. Tong, J. You, X. Zheng, Z. Xu, and X. Cheng, "Ultrafast frequency shift of electromagnetically induced transparency in terahertz metaphotonic devices," Laser Photon. Rev. 14, 1900338 (2020).

42. Y. Hu, J. You, M. Tong, X. Zheng, Z. Xu, X. Cheng, and T. Jiang, "Pump-color selective control of ultrafast all-optical switching dynamics in metaphotonic devices," Adv. Sci. 7, 2000799 (2020).

43. G. R. Yettapu, D. Talukdar, S. Sarkar, A. Swarnkar, A. Nag, P. Ghosh, and P. Mandal, "Terahertz conductivity within colloidal $\mathrm{CsPbBr}_{3}$ perovskite nanocrystals: remarkably high carrier mobilities and large diffusion lengths," Nano Lett. 16, 4838 (2016). 